Association for Information Systems

AIS Electronic Library (AISeL)

Fall 10-12-2012

Knowledge management and Semantic Technology in the Health

Care Revolution: Health 3.0 Model

Te Fu Chen Dr

Follow this and additional works at: https://aisel.aisnet.org/iceb2012

This material is brought to you by the International Conference on Electronic Business (ICEB) at AIS Electronic Library (AISeL). It has been accepted for inclusion in ICEB 2012 Proceedings by an authorized administrator of AIS Electronic Library (AISeL). For more information, please contact elibrary@aisnet.org. 


\title{
Knowledge management and Semantic Technology in the Health Care Revolution: Health 3.0 Model
}

\author{
Dr Te Fu Chen \\ Department of Business Administration, Lunghwa University of Science and Technology \\ phd2003@gmail.com
}

\begin{abstract}
Currently, the exploration, improvement, and application of knowledge management and semantic technologies to health care are in a revolution from Health 2.0 to Health 3.0. However, what accurately are knowledge management and semantic technologies and how can they improve a healthcare system? The study aims to review what constitute a Health 3.0 system, and identify key factors in the health care system. First, the study analyzes semantic web, definition of Health 2.0 and Health 3.0, new models for linked data: (1) semantic web and linked data graphs (2) semantic web and healthcare information challenges, OWL and linked knowledge, from linked data to linked knowledge, consistent knowledge representation, and Health 3.0 system. Secondly, the research analyzes two case studies of Health 3.0, and summarizes six key factors that constitute a Health 3.0 system. Finally, the study recommends the application of knowledge management and semantic technologies to Health 3.0 health care model requires the cooperation among emergency care, insurance companies, hospitals, pharmacies, government, specialists, academic researchers, and customer (patients).
\end{abstract}

Keywords: Knowledge management, Semantic Technology, Health 3.0, Healthcare, Information Technology

\section{Introduction}

Since the birth of Internet, health care providers rec-

Proceedings of the Twelfth International Conference on Electronic Business, Xi'an, China, October 12-16, 2012, 119-126. ognized the advantages of using the Internet medium to preach health information. Health 1.0 information has observably been available in an "e-pamphlet” with the approach of one-size-fits-all [14]. However, Health 1.0 has not emphasized with the user interaction and the cooperation, personalized and the use of Web 2.0 information tool though it has included the information exchange. The Health 2.0 tools are interactive and deliver personalized, more valuable information that is geared specifically to the user's input. Hence, the concept of Health 2.0 emerged [10]. People have been actively searching for more effective ways to utilize the Internet and the new movement to achieve semantic web to enhance health services, has led to the notion of Health 3.0. Pew Internet and American Life Project report that greater than $90 \%$ of young adults and nearly three quarters of all Americans access the internet on a regular basis. $80 \%$ of internet users search for health-related information [1]. Definitive evidence of health benefit from interaction with health-related virtual communities is currently lacking as further research needs to be performed [9]. Therefore, the study explores the future potential for Health 3.0.

Currently, the exploration, improvement, and application of knowledge management and semantic technologies to health care are in a revolution from Health 2.0 to Health 3.0. However, what accurately are knowledge management and semantic technologies and how can they improve a healthcare system? The study aims to review what constitute a Health 3.0 system, and identify key factors in the health care 
system.

\section{Literature Review}

\subsection{Semantic Web and health care}

Tim Berners-Lee, et al. [3] defined the Semantic Web as "a web of data that can be processed directly and indirectly by machines." The World Wide Web Consortium (W3C) promotes common formats for data on the World Wide Web, the Semantic Web is a collaborative movement [24].

Regarding semantic technology and health care, the following key points is reviewed [21]:

a. Computers can start to understand the meaning of the words and numbers that they process via semantic technologies.

b. Computers can make connections between different pieces of information that wouldn't otherwise be brought together when they have enough information from which to work.

c. This is very valuable in a health care environment, because it's simply not possible for any one medical practitioner to have enough knowledge to recognize every symptom or pattern of illness and connect it to every available cure.

d. Semantic technologies for data linking can greatly reduce the time to make the correct diagnosis and perhaps curtail the patient's uncertainty and pain from months to a few days or hours as computers can process information much faster than people.

\subsection{Semantic Web technology and Healthcare In- formation Challenges}

According to [22], the semantic web and technologies is a model for linking data, information and representing knowledge, and they will be a vital tool in solving the healthcare information challenge. They were designed accurately for such a challenge as integrating the complex and fast changing universe of health information. To get meaningful data into the system in the first place is the biggest bottleneck in the health care information equation.

Many different illnesses can present similar symptoms, and medicine is full of nuance in deciphering such complex issues with many variables. The linked data model will allow for vastly greater intelligence in the system and for the first time will enable more research on the root causes of most of the healthcare spending: the lifestyle and behavior.

In the healthcare IT debates happening on the health information technology policy committee meetings, the risks which have become apparent. And we will further enable new and better, simpler and less expensive models for representing and using the explosion of health information and knowledge. We need a new model for complex health information challenge. Semantic technology is not only a better way to link existing data, but also can start to expand horizons beyond the existing silos. [22]

\subsection{Definition of Health 2.0 and Health 3.0}

According to [10], Health 2.0 are terms representing the possibilities between health care, eHealth and Web 2.0, and has come into use after a recent spate of articles in newspapers, and by Physicians and Medical Librarians [23]; [11]. The reason for that Health has generated its own "2.0" term is its applications across health care in general, and in particular it potential in public health promotion. Crespo describes the potential as "limitless."[4]. Hughes, Joshi, Wareham [12] defines Health 2.0 as the use of a specific set of Web tools (blogs, Podcasts, tagging, search, wikis, etc) by actors in health care (doctors, patients, and scientists), using principles of open source and generation of content by users, and the power of networks in order to personalize health care, collaborate, and promote health education.

Health 3.0 is a health-related extension of the 
concept of Web 3.0 whereby the users interface with the data and information available on the web is personalized to optimize their experience [2]. Health 3.0 will utilize the data access which is based on the concept of the Semantic Web, wherein websites' data is accessible for sorting in order to tailor the presentation of information based on user preferences [3] to enable individuals to better retrieve. And contribute to personalized health-related information within networked electronic health records, and social networking resources [21]; [16]. A similar idea of semantically organizing digitized health records to create an Open Healthcare Information Architecture, was defined as health 3.0 [17]. Health 3.0 is the use of social media and incorporation of virtual tools for enhanced interactions between health care providers and consumers/patients [19].

\subsection{Goals of Health 3.0}

The main goals of health 3.0 are described as the follows:

a. Foster the creation and maintenance of supportive virtual communities within which individuals can help one another understand, cope with, and manage common health-related issues [16].

b. Increase patient self-management, preventative care and enhancing health professional expertise, facilitate an improved understanding of health issues through improved access to health related information on the web via semantic and networked resources [21]; [16].

c. Improve acceptance, understanding and adherence to best therapeutic options through personalized social networking resources [16]; [19].

d. Serve as a medium for health professionals to improve individuals' access to healthcare expertise, and to facilitate health professional-to-many-patients communication [16]; [19].

e. Digitally heals and exploits the idea of interaction through social media, in an attempt to obtain reassurance, support, and validation [13].

f. Utilize the collected information and imparted data through the web-based technologies via the expert system, connect consumers and experts via virtual reasoning tools [14].

\subsection{Health 3.0 system of USA}

According to Tony Shaw [20] the nation's health care system of USA will become increasingly digitized and semantically organized in an effort to achieve an open health care information architecture: Health 3.0, it would take shape, creating greater collaboration, visibility, and accountability in one's health care system which will ultimately lead to a healthier nation.

The digitization of the nation's health records would improve access, quality, and affordability of health care across the board as it combined with the widespread utilization of semantic technologies. The interconnected wealth of facts and resources could lead to a greatly accelerated and heightened diffusion of knowledge, a promotion of public health and preparedness, improved quality of care for all, as well as significant decreases to the general cost of health care [20].

Currently, Health 3.0 implements systems for maintaining the security of EHRs (electronic health records) and other proprietary information such as government standards like "The Health Insurance Portability and Accountability Act of 1996 (HIPAA) Privacy and Security.” [21].

\subsection{OWL and Linked Knowledge}

According to [8], the Web Ontology Language (OWL) draws from the well understood area of description logics and provides a substantially more expressive vocabulary to axiomatically describe entities for enhanced reasoning. Building these kinds of 
ontologies not only requires domain expertise to properly define describe the entities, but also requires a keen understanding of formal knowledge representation so that knowledge is properly captured and becomes intuitive to query using an information system. Several projects have now demonstrated the use of OWL-based information systems.

The HCLS knowledge base contains a collection of instantiated ontologies used to identify interesting molecular agents in the treatment of Alzheimer's [18]. With consideration of how genetics plays a role in effective drug treatment, the Pharmacogenomics Knowledge Base (PGKB) offers depression-related pharmacogenomic information that facilitates additional knowledge duration beyond the PharmGKB database [7]. Thus, ontologies can play an important role both in semantic data integration as well as guide duration activities with well established use cases towards populating a specialized knowledge base.

The integration of electronic health records with public data provides new avenues for clinical research and improved health care. With increased interest in building smarter health care systems using electronic health records, Semantic Web technologies can play a pivotal role in incentivizing interoperability between health care providers by linking valuable to public data [8].

\subsection{Consistent Knowledge Representation}

According to [8], the patterns should be coherent, intuitive and well specified such that non-experts can read, understand and apply the guidelines found there in getting users to learn about and deploy standard patterns which they can apply to their own data.. Importantly, these patterns should specify the relations that hold between instances, and for this reason having a coherent, well founded set of types and basic relations supported by formal ontology is of critical value. This necessitates significantly more effort in developing a foundational ontology (types + relations) to represent a more diverse array of knowledge, including that which is already found in linked data.

The W3C HCLS subgroup implemented a recent work on translational medicine has produced a knowledge base composed of the Translational Medicine Ontology, which provides 75 core classes mapped to 223 classes from 40 ontologies, and acts as a global schema over a set of fake patient data and linking open data (LOD) resources. They featured queries that span bedside to bench by not only matching patients to clinical trials, but also in finding trials for which their drugs had different mechanisms of action so as to potentially avoid common side effects [8].

\subsection{The model of Health 3.0 system}

The study constructs the model of Health 3.0 system through above literature review as figure 1 :

Knowledge management and semantic technology is mutual link and commonly support the Health 3.0model.

A Health 3.0 system could be utilized to create enhanced medical and clinical correlations, monitor public health, determine health practice efficacy, and conduct cost-benefit analysis of various modalities of treatments.

Knowledge Management:

a. OWL and Linked Knowledge.

b. Consistent Knowledge Representation.

Semantic technology:

a. Personalize the users interface with the data and information available on the web to optimize their experience.

b. Utilize such data access to enable individuals to better retrieve and contribute to personalized health-related information within networked electronic health records, and social networking resources. 
Health 3.0:

a. Take shape, create greater collaboration, visibility, and accountability in one's health care system.

b. Enhance interactions between healthcare providers and consumers/patient via the use of social media and incorporation of virtual tools

c. Create enhanced medical and clinical correlations, monitor public health, determine health practice efficacy, and conduct cost-benefit analysis of various modalities of treatments.

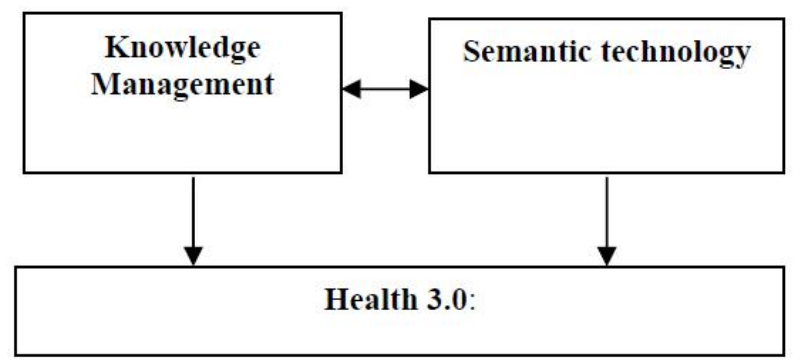

Figure 1: The model of Health 3.0 system

\section{The Case Study}

\subsection{Partners Healthcare Systems on Clinical Knowledge Management}

According to [15], Clinical Informatics Research and Development established a Clinical Knowledge Management Group in a division of Partners Healthcare System (PHS) to:

a. Implement content management infrastructure.

b. Support the management of the vast amount of knowledge encoded in clinical systems across the enterprise.

c. Leverage this knowledge via workflow portals including the EHR for care-givers and consumers, quality performance management, and clinical research.

Clinical research is separated as three phases [15]:

a. Perform an inventory of encoded knowledge assets and publish a meta-knowledge document library of the knowledge specifications for en- coded knowledge to an internally developed portal.

b. Implement Documentum eRoom and content management server solutions to support virtual, collaborative updating of decision support content as well as robust life-cycle management at the meta-knowledge level.

c. Implement new knowledge-encoding editors for ontologies and rules which they will integrate with Documentum to support life-cycle management of in-production encoded content, inheritance/propagation of content across dependent knowledge bases, and better visualization of content-interrelationships for knowledge editors and subject matter experts alike.

PHS recognizes that the arrival of personalized medicine will exponentially increase the rate of change of clinical knowledge that drives research and clinical care. The innovation adoption curve for healthcare greatly depends on a robust knowledge management infrastructure is mission to [15]:

a. Translate medicine.

b. Reduce the cost.

c. Increase the speed of knowledge discovery

d. Acquire knowledge via the clinical decision support systems.

They were going beyond classical Clinical Pathways to dynamically create a path via a decision support system, execution of procedures to create new data to calculate the next steps in the path.

\subsection{Agfa on 'Connected Knowledge'}

Knowledge is based on uniquely defined linked concepts, as a higher form of information, the Semantic Web is a way of implementing 'connected knowledge', Agfa make use of the fact that ontologies can be merged and connected to leverage existing and fragmented but highly specific pieces of knowledge, as they believe that one system cannot contain all 
medical knowledge [5].

According to [15], based on the current and constantly changing clinical information of the patient and environment, Agfa play a role of a healthcare IT vendor, they recognize the high importance of the Semantic Web to achieve their goals: a. Make systems that cross the borders of departments, hospitals, home, universities and governments. b. Give all stakeholders intelligent tools, using knowledge and guidelines, to obtain a higher quality and more cost effective health care.

\subsection{An application of Active Semantic Documents} (ASDs) in health care: Active Semantic Electronic Medical Record.

According to [5], Semantic Web technology helps achieve the goals of ASDs in an ontology driven process:

a. Reduce medical errors.

b. Improve physician efficiency.

c. Improve patient safety and satisfaction in medical practice.

This involves multiple populated ontologies, automatic semantic annotation of documents, and rule processing [5]:

a. Automatic semantic annotation of documents: ASDs are typically in XML based format of documents, and semantic, since they are semantically annotated using one or more relevant OWL ontologies which provide the nomenclature and conceptual model. They are optionally annotated using lexically significant concepts and phrases.

b. Automatic Rule processing: ASDs actively execute rules on semantic annotations and relationships that span across ontologies because they support automatic and dynamic validation and decision making on the content of the document. This Semantic rules include: (a). preven- tion of drug interaction, (b). ensuring the procedure performed has a supporting diagnosis.

Active Semantic Electronic Medical Record (ASEMR) application exemplified a practical implementation of ASDs. The abilities of ASDs are [5]:

a. Display the semantic and lexical annotations in document displaced in a browser.

b. Show results of rule execution.

c. Provide the ability to modify semantic and lexical components of its content in an ontology-supported and otherwise constrained manner.

To fix broken rules due to the above abilities to offer practical suggestions resolving the problem is time saving.

\section{Conclusions and Recommendations}

The study comprehensively reviews the related literatures of semantic technology, knowledge management, Health 2.0 and Health 3.0. Moreover, the research analyzes two case studies of Health 3.0, and identifies the following six key factors that constitute a Health 3.0 system:

1. Making publicly accessible for personal information once it has been depersonalized, and utilize to create enhanced medical and clinical correlations, monitor public health, determine health practice efficacy, and conduct cost-benefit analysis of various modalities of treatments.

2. The "health graph" has to be patient-centric. The "health graph", connecting all the participants around common data structures and standards.

3. Using artificial intelligence techniques to find out information not otherwise visible. Use a focused semantic healthcare database over a generic search engine that built-in taxonomies and a thesaurus to expand and refine the user queries.

4. Implementing a robust knowledge management infrastructure for translational medicine and content management infrastructure and support 
the management of the vast amount of knowledge encoded in clinical systems across the enterprise.

5. Establishing a decision support system, going beyond classical Clinical Pathways to dynamically create a path, and a execution of procedures to create new data to calculate the next steps in the path.

6. Making use of the fact that ontologies can be merged and connected to leverage existing and fragmented but highly specific pieces of knowledge.

Finally, the study recommends the application of semantic technologies to health care of Health 3.0 requires the cooperation among emergency care, insurance companies, hospitals, pharmacies, government, specialists, academic researchers, and customer (patients).

It will require to fully realize a considerable amount of time and legislation to achieve this cooperation. But once partnerships are formed, the open health care information architecture of Health 3.0 would be able to take shape, creating greater visibility, accountability and collaboration in one's health care system.

\section{References}

[1] Anon (2011), Pew Internet \& American Life Project. Available at: http://pewinternet.org/.

[2] Anon (2011), What is Web 3.0? Semantic Web \& other Web 3.0 Concepts Explained in Plain English.http://www.labnol.org/internet/web-3-conce pts-explained/8908/.

[3] Berners-Lee, Tim; James Hendler and Ora Lassila (May 17, 2001). "The Semantic Web". Scientific American Magazine. 284, 34-43.

[4] Crespo, R. (2007), Virtual Community Health Promotion. Preventing Chronic Disease, 4(3) : 75

[5] Dirk Colaert MD (2005), Advanced Clinical Application Manager, Agfa HealthCare "Desira- ble Features of Rule Based Systems for Medical Knowledge", Position paper for W3C workshop Rule languages for Interoperability.

[6] Dumontier, M., \& Hoehndorf, R. (2010). Realism for Scientific Ontologies. In 6th International Conference on Formal Ontology in Information Systems (p. 12). Toronto, Canada.

[7] Dumontier, M., \& Villanueva-Rosales, N. (2009). Towards pharmacogenomics knowledge discovery with the semantic web. Briefings in Bioinformatics, 10(2), 153-163.

[8] Dumontier Michel (2010), Building an effective Semantic Web for health care and the life sciences. Semantic Web 1(1-2): 131-135., IOS Press.

[9] Eysenbach G, Powell J, Englesakis M, Rizo C, Stern A. (2004), Health related virtual communities and electronic support groups: systematic review of the effects of online peer to peer interactions. BMJ. 2004;328(May):1-6

[10] Eysenbach G. (2008), Medicine 2.0: Social Networking, Collaboration, Participation, Apomediation, and Openness. J Med Internet Res. 2008; 10(3): e22. http://www.jmir.org/2008/3/ e22/.

[11] Giustini, D. (2006), How Web 2.0 is changing medicine: Editorial. British Medical Journal, 333:1283-1284

[12] Hughes B, Joshi I, Wareham J. (2008), Health 2.0 and Medicine 2.0: Tensions and Controversies in the Field, Journal of Medical Internet Research, 10(3): e23

[13] Joseph F. Coughlin (April, 2010), Health 3.0: Baby Boomers, Social Media \& the Evolution of Digital Healing, http://bigthink.com/ideas/38246? page $=$ all

[14] Marlene Beggelman (2008), Virtual Reasoning Redefining Healthcare Through Health 3.0, WHITE PAPER, Enhanced Medical Decisions 
Inc., http://www.enhancedmd.com/EMD_Virtual ReasonWP.pdf.

[15] Miller Eric (2004), Semantic Web in Health-care and Life Sciences - Applications and Demonstrations, The W3C Workshop on Semantic Web for Life Sciences, w3.org

[16] Nash, David B. (2008), "Health 3.0". P\&T 33 (2): 69-75. PMC 2730068. PMID 19749994. http://www.pubmedcentral.nih.gov/articlerender. fcgi?tool=pmcentrez\&artid=2730068. Retrieved 3 October 2011.

[17] PRWEB (May, 2010), SemTech 2010 Speaks Out About Health 3.0 -- Open Healthcare Information Architecture, http://www.prweb.com/releases/ 2010/05/prweb3957314.htm

[18] Ruttenberg, A., Clark, T., Bug, W., Samwald, M., Bodenreider, O., Chen, H., et al. (2007). Advancing translational re-search with the Semantic Web. BMC bioinformatics, 8 Supply 3, S2.

[19] Shachak, A. \& Jadad, A.R. (2010), Electronic Health Records in the Age of Social Networks and Global Telecommunications. Journal of American Medical Association, 303(5):452-453

[20] Shaw Tony (2010), Healthy knowledge: semantic technology \& the healthcare revolution. 2010, http://www.econtentmag.com/Articles/ArticleRe ader.aspx?ArticleID=69404.

[21] Shaw Tony (2010), Semantic Technology and the Health Care Revolution, 2010 Quality Digest Magazine.

[22] Steve Brown (2009), Healthcare Needs a New Information Model: Semantic Web and the Challenges in Healthcare Information Technology, http://brown2020.com/2009/07/healthcareneeds-a-new-information-model-semantic-web-a nd-the-challenges-in-healthcare-information-tec hnology/

[23] The Economist (2007), Health 2.0: Technology and society: Is the outbreak of cancer videos, bulimia blogs and other forms of "user generated" medical information a healthy trend? The Economist, September 6: 73-74

[24] W3C (2012), "XML and Semantic Web W3C Standards Timeline". 2012-02-04. http://www. dblab.ntua.gr/ bikakis/XML\%20and\%20Semant ic\%20Web\%20W3C\%20Standards\%20Timeline -History.pdf. 